Maria Helena de Sousa'

José Guilherme Cecatti'

Ellen Elizabeth Hardy'

Suzanne Jacob Serruya"

\section{Morte materna declarada e o relacionamento de sistemas de informações em saúde}

\author{
Declared maternal death and \\ the linkage between health \\ information systems
}

\section{RESUMO}

OBJETIVO: Descrever as características da mortalidade materna segundo Sistema de Informação sobre Mortalidade em relação a dados correspondentes a esses registros em outros sistemas.

MÉTODOS: Estudo descritivo, com utilização dos dois sistemas de informações de dados vitais e do sistema hospitalar, para as 26 capitais estaduais e o Distrito Federal do Brasil, em 2002. Inicialmente foram calculadas as razões de mortalidade materna e obtidas informações das mortes maternas declaradas. A partir dessas mortes relacionou-se probabilisticamente o Sistema de Informação sobre Mortalidade com o Sistema de Informações sobre Nascidos Vivos e com o Sistema de Informações Hospitalares, utilizando-se o programa "Reclink II", com estratégia de blocagem em múltiplos passos. Para os registros pareados, foram detalhados os diagnósticos e procedimentos hospitalares aproximados pelos critérios mais conhecidos de morbidade materna grave.

RESULTADOS: Foram registradas 339 mortes maternas em 2002, com razões de mortalidade materna oficial e ajustada, respectivamente, de 46,4 e 64,9 (mortes por 100.000 nascidos vivos). No relacionamento com os dados do sistema de nascidos vivos, foi possível localizar 46,5\% das mortes maternas e, com o de informações hospitalares, localizaram-se 55,2\% das mortes. O diagnóstico de internação mais freqüente foi o de infecção $(13,9 \%)$, e o procedimento com maior percentagem $(39,0 \%)$ foi o de admissão à UTI.

CONCLUSÕES: Foi baixa a percentagem de relacionamento entre os registros das três fontes estudadas. Nenhuma das possíveis falhas e/ou impossibilidade de relacionamento apontadas, isoladamente ou em conjunto, podem explicar esse baixo percentual.

Departamento de Tocoginecologi Universidade Estadual de Campinas. Campinas, SP, Brasil.

Departamento de Ciência e Tecnologia. Ministério da Saúde. Brasília, DF, Brasil.

Correspondência | Correspondence:

José Guilherme Cecatti

R. Alexander Fleming, 101

13083-881 Campinas, SP, Brasil

E-mail: cecatti@unicamp.br
DESCRITORES: Registros de Mortalidade. Mortalidade materna. Causa da morte. Estatísticas vitais. Sistemas de informações. Análise de dados.

\section{ABSTRACT}

OBJECTIVE: To describe the characteristics of maternal mortality according to the Mortality Information System in relation to the data corresponding to these records that are in other systems. 
METHODS: This was a descriptive study using two information systems on vital data and another on the hospital system, for the 26 state capitals and the Federal District of Brazil, in 2002. Initially, the maternal mortality ratios were calculated and information on declared maternal deaths were obtained. From these data, the Mortality Information System was probabilistically linked with the Live Birth Information System and the Hospital Information System, using the "Reclink II" software, with a multiple-step blocking strategy. For paired records, the diagnoses and hospital procedures brought together by the best-known criteria for severe maternal morbidity were detailed.

RESULTS: A total of 339 maternal deaths were recorded in 2002. The official and adjusted maternal mortality ratios were, respectively, 46.4 and 64.9 (deaths per 100,000 live births). By correlating with data from the live birth system, $46.5 \%$ of the maternal deaths could be located; and from the hospital information, $55.2 \%$. The most frequent admission diagnosis was infection (13.9\%) and the most frequent procedure was intensive care unit admission (39.0\%).

CONCLUSIONS: There were low percentage linkages between the records from the three sources studied. However, the possible failures and/or impossibilities in the linkages indicated may separately or jointly explain these low percentages.

\section{KEYWORDS: Mortality registries. Maternal mortality. Cause of} death. Vital statistics. Information systems. Data analysis.

\section{INTRODUÇÃO}

A mortalidade materna é difícil de ser medida, mesmo em países desenvolvidos com bom sistema de registro vital apesar do baixo sub-registro de óbitos. Pode ocorrer erro na atribuição da causa da morte, levando à subenumeração da morte materna. ${ }^{1,12}$ Mais complicada ainda é a obtenção de estimativas confiáveis nos países em desenvolvimento que, em geral, possuem baixa cobertura dos registros vitais, além de alta subenumeração de causas específicas de morte. ${ }^{1}$

O indicador de mortalidade materna mais utilizado atualmente é a razão de mortalidade materna (RMM), obtida pelo quociente entre o número de mortes maternas e o número de nascidos vivos em determinado período, multiplicado por $100.000 .{ }^{1} \mathrm{O}$ número de nascidos vivos (NV), constante de sistemas de registros vitais, é utilizado como uma aproximação ao número de mulheres grávidas, ${ }^{12}$ que idealmente deveria ser utilizado no denominador para que constituísse uma verdadeira taxa ou coeficiente. O principal problema para se calcular a RMM é a dificuldade em se identificar uma causa materna como causa do óbito claramente reconhecida e registrada, especialmente em locais onde o sistema de registro vital não existe ou é falho.

Há vários métodos para se estimar a mortalidade materna, entre eles o Reproductive Age Mortality Survey
(RAMOS) e o método das irmãs (Sisterhood method). ${ }^{8}$ Em publicação sobre estimativas da mortalidade materna em $2000,{ }^{1}$ foram apresentados os valores da RMM ajustada para 110 países, divididos em cinco blocos referentes à qualidade (em ordem decrescente) das fontes dos dados ou aos métodos utilizados para obter a RMM oficial: registro vital com boa atribuição da causa de morte, e com pobre atribuição da causa, método direto das irmãs, RAMOS e levantamento domiciliar usando estimativas diretas ou do censo.

Diversas conferências internacionais têm dado ênfase e proposto metas de redução da mortalidade materna, com prazos de alguns anos para serem alcançados. ${ }^{17}$ Dessa forma, faz-se necessária a mensuração periódica da mortalidade materna para monitoramento ao longo do tempo, apesar da dificuldade envolvida nesse processo. Recentemente surgiu interesse em se estudar a morbidade materna grave, chamada de near miss, porém ainda não existe uma definição consensual sobre esse evento. ${ }^{11,16}$

No Brasil, segundo a Pesquisa Nacional sobre Demografia e Saúde (PNDS) de 1996, a RMM foi estimada em 160 por $100.000 \mathrm{NV}$, pelo método das irmãs modificado, adotado nas pesquisas Demographic and Health Surveys (DHS).* Para o ano 2000, esse valor

\footnotetext{
* Sociedade Civil Bem-Estar Familiar no Brasil, Macro International. Pesquisa Nacional sobre Demografia e Saúde - 1996. Rio de Janeiro:
} BEMFAM; 1997. 
foi ajustado na ordem de 260 por 100.000 NV. ${ }^{1}$ Esses valores contrastam substancialmente com a RMM oficial que, em 1998, foi de aproximadamente 65 óbitos por $100.000 \mathrm{NV}$ *

O Ministério da Saúde brasileiro disponibiliza dados de seus diversos sistemas informatizados em saúde, entre eles o Sistema de Informações sobre Mortalidade (SIM), sobre Nascidos Vivos (SINASC) e sobre Informações Hospitalares (SIH). As informações do SIM estão disponíveis em meio digital desde o ano de 1979, as do SINASC desde 1991, e as do SIH compiladas e mensais, a partir de 1992 e 1994, respectivamente. ** O Ministério também divulga desde 1997, em meio impresso e na Internet, diversos indicadores básicos calculados a partir dessas várias fontes. Quanto à mortalidade materna, são apresentadas as RMM apenas para os Estados da região Sudeste (exceto Minas Gerais), Sul e Centro-Oeste (exceto Mato Grosso), devido ao fato de terem cobertura igual ou superior a $90 \%$ para o SIM e o SINASC.***

Além desses sistemas não cobrirem todo o País, a falta de enumeração completa e baixa fidedignidade das causas de morte limitam o monitoramento contínuo da mortalidade materna. ${ }^{8}$

Outro problema é que esses sistemas de rotina não são diretamente comunicáveis entre si, o que impossibilita a integração de informações parciais originadas em cada sistema num único arquivo. Essa limitação na integração entre os diferentes bancos de dados em saúde tem sido contornada com o procedimento de relacionamento probabilístico para identificar os mesmos sujeitos nestas diferentes fontes de informação. ${ }^{7}$ Assim, o objetivo do presente estudo foi descrever as características da mortalidade materna segundo sistema de informações sobre mortalidade em relação a dados correspondentes a esses registros em outros sistemas.

\section{MÉTODOS}

Estudo ecológico e descritivo, com amostra calculada de 6.932 nascidos vivos por capital para se obter as RMM. Considerou-se a RMM estimada em 65/100.000 $\mathrm{NV}$, com uma diferença absoluta de 60/100.000 NV e erro tipo I de 5\%. O tamanho da amostra foi recalculado porque quatro capitais não atingiram o número mínimo de nascidos vivos. Dessa forma, o menor número de nascidos vivos, de 3.942 em Palmas (TO), implicou na diferença absoluta de 80/100.000 NV.

Os dados do SIM, SINASC e SIH para as capitais de Estado e Distrito Federal em 2002 foram especifica- mente obtidos para o estudo, incluindo informações que permitiram identificar as mulheres. O SIH-2002 proveniente do DATASUS, no Rio de Janeiro, foi recebido em 2004, enquanto que os outros dois foram fornecidos em 2005, pela Secretaria de Vigilância em Saúde (SVS) do Ministério da Saúde, em Brasília-DF.

As variáveis consideradas de acordo com a fonte de dados, foram:

SIM: idade, raça/cor, escolaridade, estado civil e local de ocorrência do óbito;

SINASC: local de ocorrência do nascimento, número de filhos vivos, tipo de parto, número de consultas de pré-natal, peso do recém-nascido ao nascer, índice de Apgar e tempo (fontes SINASC e SIM) entre o nascimento do recém-nascido e o óbito da mãe;

SIH: diagnóstico principal e secundário, procedimento solicitado e realizado durante a internação, e total de dias de internação em unidade de terapia intensiva.

Do SIM foram selecionados inicialmente todos os registros de mulheres na faixa etária de 10 a 49 anos, cuja causa básica do óbito pertenceu à categoria "O" (capítulo XV da CID-10). Foram excluídas as categorias "O96" e "O97" referentes, respectivamente, a "morte por qualquer causa obstétrica, ocorrida entre 42 dias e um ano após o parto" (tardia) e "morte por seqüelas de causas obstétricas diretas". Do SINASC foram considerados todos os registros de nascidos vivos, enquanto que do SIH houve uma seleção inicial de mulheres de 10 a 49 anos de idade. Na seqüência, foram separados os dados das 26 capitais de Estado e do DF.

Os principais campos utilizados para o relacionamento entre dados do SIM e do SINASC foram nome e idade da mãe. Outros campos utilizados na seleção manual para confirmar o pareamento foram: data de óbito no SIM versus data de nascimento SINASC (a data no SIM deveria ser igual ou posterior à do SINASC), e endereço quando disponível. Para o pareamento entre dados do SIM e do SIH, os campos principais foram o nome e data de nascimento e os campos auxiliares foram a data de óbito da mãe no SIM versus data de saída no SIH, idade e endereço. Nos dois relacionamentos acima os campos principais foram utilizados como referência na revisão manual e confirmação dos pares verdadeiros.

O programa utilizado para o relacionamento dos sistemas foi o RECLINK II. ${ }^{2}$ Esse programa, de acesso livre, é dividido em três etapas seqüenciais: relacionamento, subdividido em blocagem e pareamento; combinação

* Ministério da Saúde. Manual dos comitês de mortalidade materna. 2. ed. Brasília: Secretaria de Políticas de Saúde, Área Técnica de Saúde da Mulher; 2002. p.7-17

** Fundação Nacional de Saúde/ Departamento de Informática do SUS. Diretório de bases de dados. Disponível em URL: <http://www. datasus.gov.br/dirbd/estrut.htm> [acesso em 16 mai 2003].

*** Rede Interagencial de Informações para a Saúde. Indicadores básicos para a saúde no Brasil: conceitos e aplicações. Brasília: Organização Pan-Americana da Saúde, 2005. Disponível em: http://tabnet.datasus.gov.br/cgi/idb2005/c03.htm [acesso em 17 jan 2007] 
dos arquivos e revisão manual. A primeira etapa, que consiste na padronização dos bancos de dados, faz-se necessária apenas uma vez, enquanto as outras duas são repetidas em vários passos, para diferentes chaves de blocagem, de acordo com o estabelecido pela avaliação subjetiva do pesquisador.

Para os dois relacionamentos (SIM x SINASC e SIM $\mathrm{x}$ SIH), aplicados separadamente em cada uma das capitais, estabeleceu-se estratégia de blocagem em múltiplos passos. ${ }^{2}$ Para o primeiro relacionamento, as chaves de blocagem foram: 1) código fonético (Soundex) do primeiro e do último nome e iniciais dos nomes intermediários; 2) código fonético do primeiro e do último nome em conjunto; 3) código fonético do primeiro nome; 4) código fonético do último nome; 5) idades iguais; 6) data de óbito no SIM igual à data de nascimento no SINASC (esta última chave foi aplicada especificamente para óbitos ocorridos na mesma data do parto).

Para o segundo relacionamento, as diferenças foram para os passos 1 e 6 , com a chave de blocagem inicial referente aos códigos fonéticos do primeiro e último nome, bem como mês e ano de nascimento. Para o sexto passo foram utilizadas as datas de nascimento iguais nos dois sistemas. Foram aplicados dois passos extras na avaliação dos bancos de dados complementares, ou seja, aqueles com registros de pessoas do sexo masculino ou com idade fora da faixa etária reprodutiva, procurandose não perder os casos inconsistentes que porventura pudessem ter sido digitados incorretamente.

A revisão manual da junção SIM versus SINASC ocorreu para os pares cujos escores foram positivos. $\mathrm{Na}$ revisão do SIM versus SIH, para o passo 1 fez-se a conferência completa, enquanto que para o passo 2 foram verificados os registros com escore total $\geq 5$. Para os passos 3 a 6 foram verificados os pares cujos escores resultaram em valor $\geq 10$. Para os dois passos extras, o escore mínimo para conferência foi igual a cinco.

A análise estatística inicial consistiu no cálculo da RMM (fontes: SIM e SINASC) oficial com respectivo intervalo de confiança (IC) de $95 \%,{ }^{6}$ e cálculo da RMM corrigida segundo fatores de ajuste obtidos por Laurenti et al, ${ }^{9} 2004$. Em seguida, realizou-se análise descritiva, por meio de tabelas de distribuição de freqüências das variáveis listadas anteriormente, para todos os casos de morte materna declarada pelo SIM e, dentre essas, as identificadas no SINASC (primeiro relacionamento) ou no SIH (segundo relacionamento). Ao final, foram descritos os diagnósticos e os procedimentos do SIH, com aproximação ao grupo de critérios de morbidade materna grave de Mantel et al, ${ }^{11} 1998$ e de Waterstone et al, ${ }^{16} 2001$, pois esses dois estudos são os mais utilizados internacionalmente para definição de morbidade materna grave. Dessa forma, esses critérios foram procurados por meio de palavras-chave na CID-10 (diagnósticos) e na lista adotada pelo Ministério da Saúde (procedimentos).

Quando mais de um registro de internação foi verificado para uma mesma pessoa, eles foram avaliados manualmente e o registro de referência foi aquele correspondente à data do óbito pelo SIM ou, quando não coincidente, o mais próximo dessa data. Dessa forma, as informações de re-internações de uma mesma mulher foram mantidas na mesma linha (registro) do banco de dados. Foram utilizados os programas Epi Info 6.04d e o SPSS v. 11.5.

O estudo foi aprovado pelo Comitê de Ética em Pesquisa da Faculdade de Ciências Médicas da Universidade Estadual de Campinas (Parecer n ${ }^{\circ}$ 147/2004) e segue os princípios enunciados na Declaração de Helsinque. Foi garantido o sigilo da identificação dos casos.

\section{RESULTADOS}

Em 2002, para o total das capitais de Estado, foram registradas 339 mortes maternas com causa básica no SIM, e 730.800 nascidos vivos no SINASC, resultando em RMM oficial de 46,4/100.000 NV. Os valores mínimo e máximo para as RMM ocorreram, respectivamente, em Florianópolis (onde não foi registrado óbito materno) e em Palmas com valor de 126,8/100.000 NV. As capitais da região Norte tiveram as maiores amplitudes dos intervalos de confiança, implicando em menor precisão, em especial devido ao menor número de nascidos vivos e ao pequeno número absoluto de mortes maternas. Utilizando-se fatores de ajuste por região foram obtidas as RMM ajustadas, sendo que para o total das capitais a estimativa foi de 64,9/100.000 NV (Tabela 1).

Das 339 mortes maternas registradas no SIM, 74,3\% foram de mulheres com menos de 35 anos e $97 \%$ ocorreram em hospital. Para aquelas mulheres cuja informação estava disponível no SIM, 47\% eram pardas e 39\% brancas, $61 \%$ tinham até sete anos de escolaridade, e $58 \%$ eram solteiras. Dessas três variáveis a escolaridade teve maior proporção (35\%) de informação ignorada (Tabela 2).

Com o relacionamento do SIM com o SINASC foi possível identificar apenas 46,5\% $(\mathrm{N}=140)$ das 301 mortes maternas, já excluídas do total aquelas decorrentes de complicações de aborto. Dessa forma, as variáveis reprodutivas dessas 140 mulheres foram descritas, sendo que $99,3 \%$ tiveram o parto em hospital, dos quais $65 \%$ por cesárea. Para as mulheres cuja informação estava disponível no SINASC, aproximadamente 52\% tinham até um filho vivo, $47 \%$ haviam passado por mais de seis consultas de pré-natal e $41 \%$ tinham parido um bebê com peso menor que $2.500 \mathrm{~g}$. O tempo entre a data do nascimento da criança e a data de óbito da mãe foi de até dez dias em $73 \%$ dos 138 registros (Tabela 3 ). 
Tabela 1. Número de mortes maternas, nascidos vivos, razão de mortalidade materna oficial e respectivo intervalo de confiança e ajustada para as capitais de estado, Brasil, 2002.

\begin{tabular}{|c|c|c|c|c|c|c|}
\hline Capital & MM & NV & $\begin{array}{l}\text { RMM } \\
\text { oficial }\end{array}$ & $\begin{array}{c}\text { IC } 95 \% \\
\text { para } \\
\text { RMM }\end{array}$ & Fator de ajuste* & $\begin{array}{c}\text { RMM } \\
\text { ajustada }\end{array}$ \\
\hline Região Norte & 54 & 97.461 & 55,4 & {$[42,0 ; 72,9]$} & 1,08 & 59,8 \\
\hline Porto Velho & 3 & 7.202 & 41,7 & {$[10,8 ; 132,7]$} & & 45,0 \\
\hline Rio Branco & 2 & 7.710 & 25,9 & {$[4,5 ; 104,6]$} & & 28,0 \\
\hline Manaus & 23 & 38.161 & 60,3 & {$[39,1 ; 92,0]$} & & 65,1 \\
\hline Boa Vista & 1 & 6.072 & 16,5 & {$[0,9 ; 106,9]$} & & 17,8 \\
\hline Belém & 13 & 25.795 & 50,4 & {$[28,0 ; 88,7]$} & & 54,4 \\
\hline Macapá & 7 & 8.795 & 81,6 & {$[35,8 ; 176,2]$} & & 88,1 \\
\hline Palmas & 5 & 3.942 & 126,8 & {$[46,7 ; 314,2]$} & & 137,0 \\
\hline Região Nordeste & 101 & 187.146 & 54,0 & {$[44,2 ; 65,9]$} & 1,76 & 95,0 \\
\hline São Luís & 14 & 18.317 & 76,4 & {$[43,5 ; 131,7]$} & & 134,5 \\
\hline Teresina & 5 & 14.498 & 34,5 & {$[12,7 ; 85,5]$} & & 60,7 \\
\hline Fortaleza & 19 & 39.301 & 48,3 & {$[30,0 ; 77,1]$} & & 85,1 \\
\hline Natal & 6 & 13.286 & 45,2 & {$[18,4 ; 103,6]$} & & 79,5 \\
\hline João Pessoa & 8 & 11.140 & 71,8 & {$[33,4 ; 147,6]$} & & 126,4 \\
\hline Recife & 15 & 24.307 & 61,7 & {$[35,9 ; 104,4]$} & & 108,6 \\
\hline Maceió & 7 & 16.599 & 42,2 & {$[18,5 ; 91,1]$} & & 74,2 \\
\hline Aracajú & 6 & 9.354 & 64,1 & {$[26,1 ; 147,2]$} & & 112,9 \\
\hline Salvador & 21 & 40.344 & 52,1 & {$[33,1 ; 81,1]$} & & 91,6 \\
\hline Região Sudeste & 131 & 307.408 & 42,6 & {$[35,8 ; 50,7]$} & 1,35 & 57,5 \\
\hline Belo Horizonte & 10 & 32.601 & 30,7 & {$[15,6 ; 58,4]$} & & 41,4 \\
\hline Vitória & 1 & 4.444 & 22,5 & {$[1,2 ; 146,0]$} & & 30,4 \\
\hline Rio de Janeiro & 52 & 86.949 & 59,8 & {$[45,1 ; 79,1]$} & & 80,7 \\
\hline São Paulo & 68 & 183.414 & 37,1 & {$[29,0 ; 47,3]$} & & 50,1 \\
\hline Região Sul & 18 & 51.649 & 34,9 & {$[21,3 ; 56,3]$} & 1,83 & 63,8 \\
\hline Curitiba & 7 & 26.371 & 26,5 & {$[11,6 ; 57,3]$} & & 48,6 \\
\hline Florianópolis & 0 & 5.229 & 0,0 & {$[0,0 ; 91,5]$} & & 0,0 \\
\hline Porto Alegre & 11 & 20.049 & 54,9 & {$[28,9 ; 101,4]$} & & 100,4 \\
\hline Região Centro-Oeste & 35 & 87.136 & 40,2 & {$[28,4 ; 56,5]$} & 1,10 & 44,2 \\
\hline Campo Grande & 4 & 12.347 & 32,4 & {$[28,4 ; 56,5]$} & & 35,6 \\
\hline Cuiabá & 7 & 8.953 & 78,2 & {$[34,3 ; 168,8]$} & & 86,0 \\
\hline Goiânia & 8 & 20.037 & 39,9 & {$[18,6 ; 82,1]$} & & 43,9 \\
\hline Brasília & 16 & 45.799 & 34,9 & {$[20,7 ; 58,1]$} & & 38,4 \\
\hline Total das Capitais & 339 & 730.800 & 46,4 & {$[41,6 ; 51,7]$} & 1,40 & 64,9 \\
\hline
\end{tabular}

Fonte: Laurenti et al ${ }^{9}(2004)$

MM: Morte materna pelo Sistema de Informação sobre Mortalidade (SIM), pela causa básica, exceto subgrupos O96 e O97.

NV: Nascidos vivos, pelo Sistema Informação sobre Nascidos Vivos (SINASC).

RMM: razão de mortalidade materna, por $100.000 \mathrm{NV}$;

A partir dos dados do SIM, foi possível localizar no SIH $55,2 \%(\mathrm{~N}=187)$ das 339 mortes maternas declaradas. Desses 187 registros, as percentagens obtidas para os diversos tipos de diagnósticos foram de no máximo $13,9 \%$, referente a problemas relativos à infecção, seguida por problemas cardíacos $(10,7 \%)$. Para os procedimentos, a maior percentagem foi para o item admissão à Unidade de Terapia Intensiva (UTI) (39,0\%), seguida por problemas cardíacos, $11,8 \%$, e de infecção $10,7 \%$ (Tabela 4). Avaliando-se os diagnósticos e pro- 
Tabela 2. Distribuição percentual dos registros de morte materna declarada nas capitais estaduais, segundo variáveis sociodemográficas. Brasil, 2002. N=339

\begin{tabular}{|c|c|c|}
\hline Variável & $\mathrm{N}$ & $\%$ \\
\hline \multicolumn{3}{|l|}{ Idade (anos) } \\
\hline$\leq 19$ & 40 & 11,8 \\
\hline $20-24$ & 63 & 18,6 \\
\hline $25-29$ & 77 & 22,7 \\
\hline $30-34$ & 72 & 21,2 \\
\hline $35-39$ & 69 & 20,4 \\
\hline$\geq 40$ & 18 & 5,3 \\
\hline \multicolumn{3}{|l|}{ Raça (cor) $[\mathrm{N}=304(\mathrm{a})]$} \\
\hline Branca & 119 & 39,1 \\
\hline Preta & 41 & 13,5 \\
\hline Parda & 143 & 47,0 \\
\hline Indígena & 1 & 0,3 \\
\hline \multicolumn{3}{|l|}{ Escolaridade [N=219 (b)] } \\
\hline Nenhuma & 12 & 5,5 \\
\hline $1-3$ anos & 44 & 20,1 \\
\hline $4-7$ anos & 78 & 35,6 \\
\hline$\geq 12$ anos & 25 & 11,4 \\
\hline \multicolumn{3}{|l|}{ Estado civil [N=313 (c)] } \\
\hline Solteira & 182 & 58,1 \\
\hline Casada & 101 & 32,3 \\
\hline Viúva & 1 & 0,3 \\
\hline Separada judicialmente & 5 & 1,6 \\
\hline União consensual & 24 & 7,7 \\
\hline \multicolumn{3}{|l|}{ Local de ocorrência do óbito } \\
\hline Hospital & 328 & 96,8 \\
\hline Via pública & 1 & 0,3 \\
\hline Domicílio & 6 & 1,8 \\
\hline Outro & 4 & 1,2 \\
\hline
\end{tabular}

Fonte: SIM, 2002.

Percentagem de ignorados: (a) 10,3\%; (b) $35,4 \%$; (c) $7,7 \%$.

cedimentos em conjunto, houve $66 \%$ de registros com relato de pelo menos uma condição mórbida materna (dado não apresentado em tabela).

\section{DISCUSSÃO}

Nem sempre é fácil reconhecer quando a morte de uma mulher em idade fértil ocorreu devido a uma causa materna. Vários fatores podem influenciar o correto preenchimento da declaração de óbito: assistência médica no momento do óbito, tipo de serviço (emergência, UTI, obstetrícia, clínica médica), profissional que prestou a assistência e quem preencheu a declaração de óbito. O diagnóstico da causa básica de morte materna
Tabela 3. Distribuição percentual* dos registros de morte materna declarada nas capitais estaduais, encontrados no Sistema de Informação de Nascidos Vivos, segundo variáveis. Brasil, 2002. $\mathrm{N}=140$ (46,5\% de 301 mortes maternas**)

\begin{tabular}{|c|c|c|}
\hline Variável & $\mathrm{N}$ & $\%$ \\
\hline \multicolumn{3}{|c|}{ Local de ocorrência do nascimento } \\
\hline Hospital & 139 & 99,3 \\
\hline Domicílio & 1 & 0,7 \\
\hline \multicolumn{3}{|c|}{ Número de filhos vivos $[\mathrm{N}=114$ (a)] } \\
\hline Nenhum & 31 & 27,2 \\
\hline 1 & 28 & 24,6 \\
\hline 2 & 27 & 23,7 \\
\hline$\geq 3$ & 28 & 24,6 \\
\hline \multicolumn{3}{|l|}{ Tipo de parto } \\
\hline Vaginal & 49 & 35,0 \\
\hline Cesárea & 91 & 65,0 \\
\hline \multicolumn{3}{|c|}{ Número de consultas de pré-natal [N=131 (b)] } \\
\hline Nenhuma & 5 & 3,8 \\
\hline 1 a 3 & 15 & 11,5 \\
\hline 4 a 6 & 49 & 37,4 \\
\hline$\geq 7$ & 62 & 47,3 \\
\hline \multicolumn{3}{|c|}{ Peso ao nascer (g) [N=138 (c)] } \\
\hline$<1.500$ & 15 & 10,9 \\
\hline $1.500-2.499$ & 41 & 29,7 \\
\hline $2.500-3.499$ & 61 & 44,2 \\
\hline$\geq 3.500$ & 21 & 15,2 \\
\hline \multicolumn{3}{|c|}{ Índice de Apgar [N=134 (d)] } \\
\hline$<7$ ao $1^{\circ}$ minuto & 54 & 40,3 \\
\hline$<7$ ao $5^{\circ}$ minuto & 26 & 19,4 \\
\hline
\end{tabular}

Tempo entre nascimento do RN e óbito da mãe (dias)*** $[\mathrm{N}=138$ (c)]

\begin{tabular}{lll}
0 & 31 & 22,5 \\
1 & 18 & 13,0 \\
2 a 5 & 26 & 18,8 \\
11 a 19 & 14 & 10,1 \\
$\geq 20$ & 23 & 16,7 \\
\hline
\end{tabular}

Fonte: SINASC, 2002

* Para o índice de Apgar apresentou-se a percentagem $(<7)$ ao $1^{\circ}$ e $5^{\circ}$ minuto

** Exceto mortes maternas por aborto: 38

*** Fontes: SIM e SINASC

Percentagem de ignorados: (a) $18,6 \%$; (b) $6,4 \%$; (c) $1,4 \%$; (d) $4,3 \%$

torna-se mais difícil quando o óbito ocorre no início da gravidez ou no puerpério, ou se a causa da morte foi indireta, especialmente se não houvesse conhecimento prévio da gravidez.

Estudos realizados no Brasi $1^{3,15}$ mostram que em função dessas situações, a atribuição da causa básica do 
Tabela 4. Percentagem dos registros de morte materna declarada das capitais estaduais encontrados no Sistema de Informação Hospitalar, ${ }^{*}$ segundo problema ou disfunção, para diagnósticos e procedimentos. Brasil, 2002. $\mathrm{N}=187$ (55,2\% de 339 mortes maternas)

\begin{tabular}{|c|c|c|}
\hline $\begin{array}{l}\text { Problema ou disfunção, } \\
\text { ou manejo }\end{array}$ & Diagnósticos & Procedimentos \\
\hline \multicolumn{3}{|c|}{ Aproximação aos critérios de Mantel et al ${ }^{11}$} \\
\hline Cardíaco & 10,7 & 11,8 \\
\hline Vascular & 0,5 & - \\
\hline $\begin{array}{l}\text { Imunológico (septi- } \\
\text { cemia, infecção) }\end{array}$ & 13,9 & 10,7 \\
\hline Respiratório & 4,8 & 5,3 \\
\hline Renal & 1,6 & 0,5 \\
\hline Do fígado & 0,0 & 0,0 \\
\hline Metabólico & 0,5 & 0,5 \\
\hline De coagulação & 0,5 & 0,5 \\
\hline Cerebral & 2,1 & 1,1 \\
\hline Admissão à UTI & - & $39,0^{* *}$ \\
\hline $\begin{array}{l}\text { Histerectomia de } \\
\text { emergência }\end{array}$ & - & 5,9 \\
\hline $\begin{array}{l}\text { Acidentes anesté- } \\
\text { sicos }\end{array}$ & 0,0 & - \\
\hline \multicolumn{3}{|c|}{ Aproximação aos critérios de Waterstone et al ${ }^{16}$} \\
\hline Pré-eclâmpsia grave & 4,3 & 3,2 \\
\hline Eclâmpsia & 7,0 & 2,7 \\
\hline Síndrome HELLP & - & - \\
\hline Hemorragia grave & 5,9 & 2,1 \\
\hline Sepse grave & 13,9 & 10,7 \\
\hline Ruptura uterina & 0,0 & - \\
\hline \multicolumn{3}{|l|}{ Outros critérios } \\
\hline Abdome agudo & 3,2 & - \\
\hline Morte obstrética & 0,5 & - \\
\hline \multicolumn{3}{|c|}{$\begin{array}{l}\text { Fonte: SIH } \\
\text { HELLP: complicação grave da pré-eclâmpsia, caracte- } \\
\text { rizada por hemólise, elevação das enzimas hepáticas e } \\
\text { plaquetopenia. } \\
\text { * Entre todas as internações de mulheres de } 10 \text { a } 49 \\
\text { anos em } 2002 \text { nas capitais brasileiras (SIH, 2002) } \\
\text { ** Não encontrado entre os seis procedimentos de } \\
\text { diária de unidade de terapia intensiva (UTI) localizado } \\
\text { no campo referente ao total de dias de UTI durante a } \\
\text { internação. } \\
\text { - Sem códigos relacionados ao problema }\end{array}$} \\
\hline
\end{tabular}

óbito e a mensuração da mortalidade materna ficam em geral subestimadas. De fato, recente publicação da $\mathrm{OMS}^{18}$ mostrou subestimação da RMM no Estado do Paraná, após investigação dos casos pelos comitês de morte materna.

Para se utilizar a RMM oficial como indicador válido, de forma contínua, especialmente para avaliação do alcance de metas preconizadas de redução da mortalidade materna,${ }^{17}$ faz-se necessário conscientizar os profissionais responsáveis pelo preenchimento da declaração de óbito. Eles devem ser treinados para buscar informações detalhadas e investigar melhor a condição que precedeu o óbito de uma mulher em idade reprodutiva. Isso melhoraria a qualidade da informação em saúde, com maior validade e precisão dos indicadores.

Outra questão importante para obtenção da RMM é saber se os dados oficiais nacionais possuem alta cobertura dos eventos vitais nos vários sistemas existentes, pois tanto o numerador quanto o denominador podem estar incorretos. No Brasil, o Ministério da Saúde tem divulgado a RMM apenas para os Estados da região Sudeste, Sul e Centro-Oeste (exceto Mato Grosso), com cobertura mínima de $90 \%$ para os sistemas de informações vitais. Dessa forma, grande parte do território brasileiro não possui boa cobertura dos eventos vitais, apesar da gratuidade para obtenção da declaração de óbito.

Em função do sub-registro de óbitos e subenumeração de causas maternas, pode-se questionar a validade e confiabilidade dos valores oficiais da RMM. Observou-se no presente estudo que, apesar de terem sido analisadas apenas as capitais, o valor corrigido da RMM foi de 65/100.000 NV. Esse valor foi igual ao divulgado pelo Ministério da Saúde em 1998, e diferente do obtido pela PNDS em 1996 e da estimativa da OMS para o ano $2000,{ }^{1}$ esses dois últimos respectivamente de 160 e 260/100.000 nascidos vivos. Pensando-se apenas em ordens de grandeza, dado que as populações, os períodos e os métodos foram diferentes pode-se imaginar que a qualidade da informação não melhorou substancialmente nos últimos anos.

O foco principal do presente estudo foi testar a utilização dos bancos de dados de informações rotineiras em saúde como fonte principal desta informação. A correção das RMM pelos fatores de ajuste por região do País ${ }^{8}$ pode levar a super ou subestimativas em cada capital dentro de uma mesma região. Entretanto, julgou-se pertinente utilizar esses fatores, pois são utilizados pelo próprio Ministério da Saúde para fazer a correção da mortalidade materna, obtida segundo as declarações de óbito.

No Brasil, os sistemas informatizados de dados vitais (SIM e SINASC), bem como de dados hospitalares (SIH), apresentam informações inconsistentes. Considerando que mais de $96 \%$ das mortes maternas declaradas tiveram registrado o hospital como local de ocorrência do óbito, a percentagem de registros encontrados em dois relacionamentos no presente estudo foi insatisfatória (SIM x SINASC com 46,5\%, e SIM $\mathrm{x}$ SIH com 55,2\%).

Pode-se imaginar, ainda, que algumas mortes maternas tenham ocorrido em hospitais privados, sem vínculo 
com o Sistema Único de Saúde. Porém, não seria esperado que esse fato fosse relevante para explicar a não localização de pares SIM versus SIH.

Em publicação sobre o relacionamento probabilístico de um levantamento domiciliar com os dados do SIH, Coeli et al, ${ }^{5}$ encontraram baixa percentagem de pareamento entre os registros das duas fontes. Destacaram entre os possíveis erros no processo de relacionamento, a utilização de vários campos em vez de um identificador único (inexistente), e levantaram a possibilidade de que tenha ocorrido registro incompleto das autorizações de internação hospitalar (AIH), por problemas administrativos ou cobertura parcial dos serviços.

Quanto ao relacionamento SIM x SINASC, parte dos registros não encontrados provavelmente deveu-se a casos de natimortos e de gravidez ectópica, além dos abortos inicialmente identificados. Outras possíveis explicações seriam: a ocorrência de partos domiciliares não registrados oficialmente, de óbitos sem internação hospitalar e de possíveis internações sem emissão do correspondente documento para inserção no SIH.

Apesar do exposto sobre as possíveis falhas e/ou impossibilidade de pareamento dos registros, nenhuma destas situações alternativas, isoladamente ou em conjunto, consegue explicar um percentual tão baixo de identificação de casos no relacionamento entre os sistemas. Isso porque os eventos (morte materna) são relativamente de baixa prevalência e porque as informações são apenas das capitais, onde os dados costumam ser de melhor qualidade. É possível que parte desses registros não encontrados seja referente a nascimento ou internação

\section{REFERÊNCIAS}

1. AbouZahr C, Wardlaw T. Maternal mortality in 2000: estimates developed by WHO, Unicef and UNFPA. Geneva: WHO; 2003.

2. Camargo Jr KR, Coeli CM. Reclink: aplicativo para o relacionamento de bases de dados, implementando o método probabilistic record linkage. Cad Saúde Pública. 2000;16(2):439-47.

3. Cecatti JG, Faúndes A, Surita FGC. Maternal mortality in Campinas: evolution, under-registration and avoidance. São Paulo Med J. 1999;117(1):5-12.

4. Coeli CM, Camargo Jr KR. Avaliação de diferentes estratégias de blocagem no relacionamento probabilístico de registros. Rev Bras Epidemiol. 2002;5(2):185-96.

5. Coeli CM, Costa MCE, Almeida LM. Probabilistic linkage in household survey on hospital care usage. Rev Saúde Pública. 2003;37(1):91-9.

6. Fleiss JL. Statistical methods for rates and proportions. $2^{\text {nd }}$ ed. New York: John Wiley and Sons; 1981.

7. Jaro MA. Advances in record-linkage methodology as applied to matching the 1985 Census of Tampa, ocorridos em 2001 e, desta forma, foram perdidos por não se ter ampliado retrospectivamente o período de análise. Entretanto esse processo não foi feito devido especialmente à inviabilidade operacional.

A experiência brasileira em relacionamento probabilístico de bases de dados é recente, tendo como ponto de partida o desenvolvimento do programa Reclink, que está em sua segunda versão e é gratuito. ${ }^{2}$ Com isso, têm sido feitos estudos metodológicos com o intuito de avaliar e melhorar as estratégias empregadas no relacionamento de bancos de dados em saúde. ${ }^{4,10}$

Observou-se que a maioria dos óbitos localizados no SINASC tinha ocorrido nos primeiros dez dias após o parto. Também, dois terços dos registros do SIM encontrados no SIH tiveram diagnósticos e procedimentos relativos a pelo menos uma condição mórbida materna grave, que talvez pudesse ser explorada como um preditor da morte materna. Em revisões sobre morte materna e morbidade materna grave, ${ }^{13,14}$ sugere-se que mulheres que foram a óbito ou que sofreram sérios agravos associados à gravidez, poderiam ter características semelhantes, de acordo com alguns estudos publicados. ${ }^{11,16}$

Estudos futuros baseados em registros hospitalares poderão explorar melhor as situações de mortalidade e morbidade materna grave. Além disso, o tema de relacionamento de bancos de dados, relativamente novo no Brasil, implica em promissora perspectiva de se explorar conjuntamente as diversas fontes de informação em saúde.
Florida. J Am Stat Assoc. 1989;84:414-20.

8. Laurenti R, Mello-Jorge MHP, Gotlieb SLD. Reflexões sobre a mensuração da mortalidade materna. Cad Saúde Pública. 2000;16(1):23-30.

9. Laurenti R, Mello-Jorge MHP, Gotlieb SLD. A mortalidade materna nas capitais brasileiras: algumas características e estimativa de um fator de ajuste. Rev Bras Epidemiol. 2004;7(4):449-60.

10. Machado CJ, Hill K. Probabilistic record linkage and an automated procedure to minimize the undecided-matched pair problem. Cad Saúde Pública. 2004;20(4):915-25

11. Mantel GD, Buchmann E, Rees H, Pattinson RC. Severe acute maternal morbidity: a pilot study of a definition for a near-miss. Br J Obstet Gynaecol. 1998;105(9):985-90.

12. Royston E, AbouZahr C. Measuring maternal mortality. Br J Obstet Gynaecol. 1992;99:540-2.

13. Sousa MH, Cecatti JG, Hardy EE, Amaral E, Souza JPD, Serruya S. Sistemas de informação em saúde 
e o monitoramento de morbidade materna grave e mortalidade materna. Rev Bras Saúde Matern Infant. 2006;6(2):161-8.

14. Souza JPD, Cecatti JG, Parpinelli MA, Sousa $\mathrm{MH}$, Serruya S. Revisão sistemática sobre morbidade materna near miss. Cad Saúde Pública. 2006;22(2):255-64.

15. Theme Filha MM, Silva RI, Noronha CP. Mortalidade materna no município do Rio de Janeiro, 1993-1996. Cad Saúde Pública. 1999;15(2):397-403.
16. Waterstone $\mathrm{M}$, Bewley $\mathrm{S}$, Wolfe $\mathrm{C}$. Incidence and predictors of severe obstetric morbidity: case-control study. BMJ. 2001;322(7294):1089-93.

17. World Health Organization. Reduction of maternal mortality: a joint WHO/UNFPA/Unicef World Bank Statement. Geneva; 1999.

18. World Health Organization. Beyond the numbers: reviewing maternal deaths and complications to make pregnancy safer. Geneva; 2004. 